\title{
Herschel-SPIRE FTS spectroscopy of the carbon-rich objects AFGL 2688, AFGL 618, and NGC 7027 $7^{\star, \star \star}$
}

\author{
R. Wesson ${ }^{1}$, J. Cernicharo ${ }^{2}$, M. J. Barlow ${ }^{1}$, M. Matsuura ${ }^{1,3}$, L. Decin ${ }^{4,5}$, M. A. T. Groenewegen ${ }^{6}$, E. T. Polehampton ${ }^{7,8}$, \\ M. Agundez ${ }^{9}$, M. Cohen ${ }^{10}$, F. Daniel ${ }^{2}$, K. M. Exter ${ }^{4}$, W. K. Gear ${ }^{11}$, H. L. Gomez ${ }^{11}$, P. C. Hargrave ${ }^{11}$, P. Imhof ${ }^{12}$, \\ R. J. Ivison ${ }^{13}$, S. J. Leeks ${ }^{7}$, T. L. Lim ${ }^{7}$, G. Olofsson ${ }^{14}$, G. Savini ${ }^{1}$, B. Sibthorpe ${ }^{13}$, B. M. Swinyard ${ }^{7}$, T. Ueta ${ }^{15}$, \\ D. K. Witherick ${ }^{1}$, and J. A. Yates ${ }^{1}$
}

(Affiliations are available in the online edition)

Received 30 March 2010 / Accepted 10 May 2010

\section{ABSTRACT}

We present far-infrared and submillimetre spectra of three carbon-rich evolved objects, AFGL 2688, AFGL 618 and NGC 7027. The spectra were obtained with the SPIRE Fourier-transform spectrometer on board the Herschel Space Observatory, and cover wavelengths from $195-670 \mu$ m, a region of the electromagnetic spectrum hitherto difficult to study in detail. The far infrared spectra of these objects are rich and complex, and we measure over 150 lines in each object. Lines due to 18 different species are detected. We determine physical conditions from observations of the rotational lines of several molecules, and present initial large velocity gradient models for AFGL 618. We detect water in AFGL 2688 for the first time, and confirm its presence in AFGL 618 in both ortho and para forms. In addition, we report the detection of the $J=1-0$ line of $\mathrm{CH}^{+}$in NGC 7027.

Key words. astrochemistry - line: identification - circumstellar matter - stars: evolution - stars: general - infrared: stars

\section{Introduction}

Low- to intermediate-mass stars $\left(<8 M_{\odot}\right)$ shed much of their mass during the final stages of their evolution, in the form of a slow molecular wind. The mass loss causes the star to leave the asymptotic giant branch (AGB), moving to the left in the Hertzsprung-Russell diagram. The star becomes hotter, warming and eventually ionising its previously ejected outer layers, which become visible as a planetary nebula (PN). The ejecta from such stars are a major contributor to galactic chemical evolution (e.g. Matsuura et al. 2009).

The evolution from the AGB to the PN stage is rapid, and relatively few objects in this intermediate stage are known (326 are listed by Szczerba et al. 2007, compared to around 3000 known or suspected PNe Frew \& Parker 2010). AFGL 618 and AFGL 2688 (the Egg Nebula) are two of the best-known objects in this transition stage. AFGL 2688 is illuminated by a central star with spectral type F5, and Hubble Space Telescope images reveal a large number of round arcs crossed by "searchlight beams" (Sahai et al. 1998). Proper motion measurements give a distance of $420 \mathrm{pc}$ and a dynamical age for the ejected material of 350 years (Ueta et al. 2006). AFGL 618 entered the protoplanetary nebula (PPN) phase 100-200 years ago (Kwok \& Bignell 1984; Bujarrabal et al. 1988), and is more evolved than AFGL 2688. It contains a B0 central star surrounded by a compact H II region (Wynn-Williams 1977; Kwok \& Bignell 1984). The variability of free-free emission from this object

* Herschel is an ESA space observatory with science instruments provided by European-led Principal Investigator consortia and with important participation from NASA.

$\star \star$ Tables 1-4 are only available in electronic form at http://www . aanda.org over the last 30 years implies rapid evolution (Kwok \& Feldman 1981; Sánchez Contreras et al. 2002). NGC 7027 is a very young planetary nebula (Masson 1989). It has been extensively studied due to its high surface brightness at all wavelengths, and observations with the ISO revealed a far-infrared spectrum rich in atomic and molecular lines (Liu et al. 1996). Its ionised inner regions are surrounded by and partly obscured by a PDR and massive molecular envelope.

Previous far infrared and sub-mm observations of these evolving post-AGB objects, although limited in their spatial resolution and spectral coverage, have revealed rich spectra containing numerous molecular and dust features (e.g. Cox et al. 1996; Liu et al. 1996; Herpin et al. 2000; Pardo et al. 2004, 2005; Pardo \& Cernicharo 2007; Pardo et al. 2007). The Herschel Space Observatory (Pilbratt et al. 2010) now significantly extends our observational capabilities. The SPIRE spectrometer covers wavelengths from $195-670 \mu \mathrm{m}$, a region of the electromagnetic spectrum hitherto largely unexplored. The SPIRE instrument, its in-orbit performance, and its scientific capabilities are described by Griffin et al. (2010), and the SPIRE astronomical calibration methods and accuracy are outlined by Swinyard et al. (2010). Here we present observations of AFGL 2688, AFGL 618 and NGC 7027 using the SPIRE spectrometer.

\section{Observations}

Observations of AFGL 2688, AFGL 618 and NGC 7027 were obtained using the SPIRE Fourier-transform spectrometer, which covers short (SSW, 194-313 $\mu \mathrm{m}$ ) and long (SLW, 303-671 $\mu \mathrm{m}$ ) wavelength bands simultaneously. We combine data taken during the satellite's performance verification phase with data taken during the science demonstration phase for the 

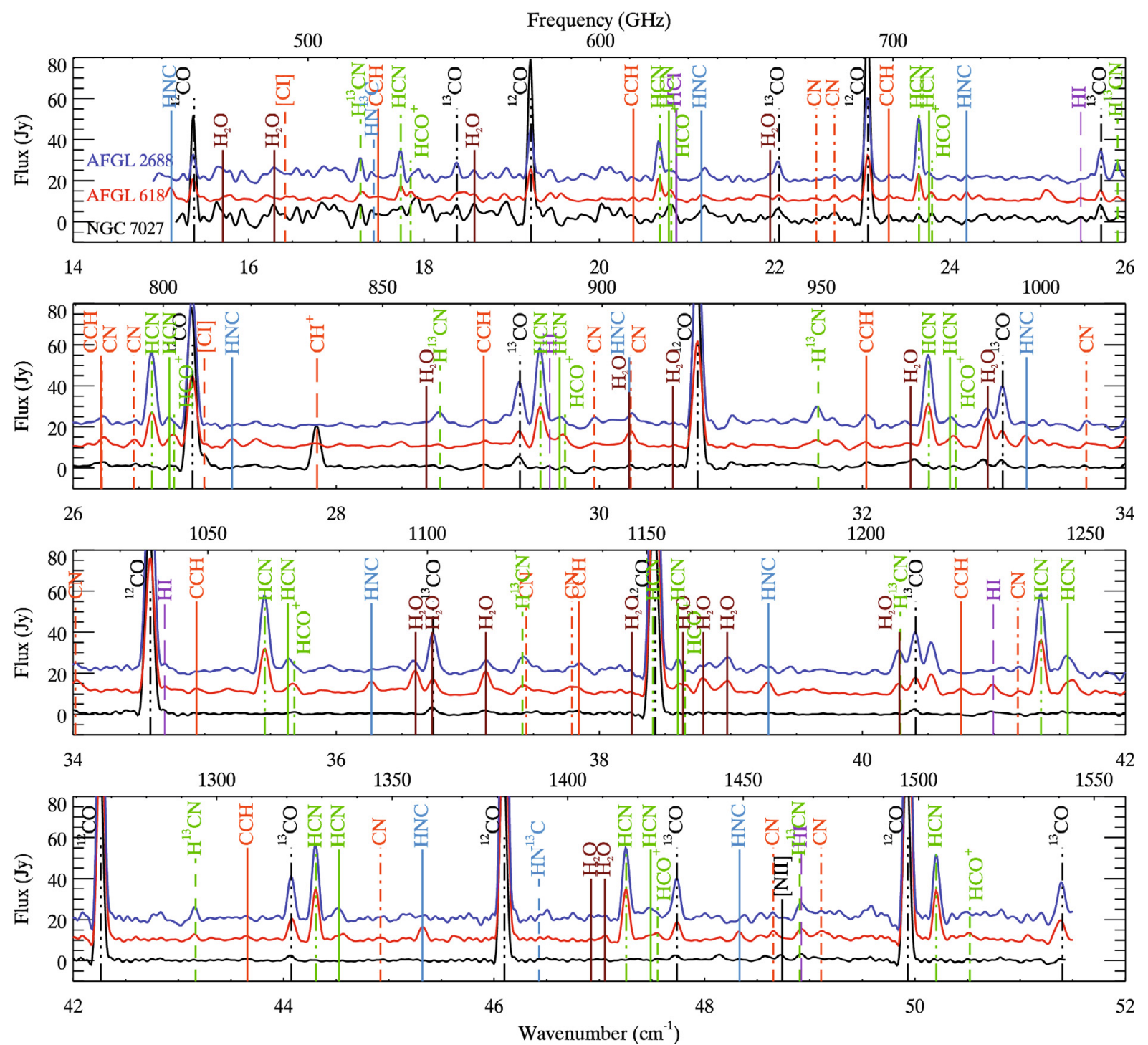

Fig. 1. Continuum-subtracted SPIRE FTS spectra of NGC 7027 (black), AFGL 618 (red) and AFGL 2688 (blue). For clarity, the spectrum of NGC 7027 is multiplied by a factor of two, the spectrum of AFGL 618 is offset by $10 \mathrm{Jy}$, and the spectrum of AFGL 2688 is offset by 20 Jy.

Mass-loss of Evolved StarS (MESS ${ }^{1}$ ) guaranteed time key program (Groenewegen et al., in prep.). We reduced the spectra with the Herschel interactive processing environment software (HIPE), using the standard point-source pipeline described by Swinyard et al. (2010). A dark-sky observation taken close to the time of the object observations was subtracted to correct for telescope and sky emission, and the spectra were flux-calibrated using observations of the asteroid Vesta. We combined three observations of AFGL 618, five of AFGL 2688 and seven of NGC 7027 to obtain our final spectra. The total on-source exposure times were 11988s (AFGL 618), $4928 \mathrm{~s}$ (AFGL 2688) and $11188 \mathrm{~s}$ (NGC 7027).

The FTS produces spectra in which the line profiles are sinc functions; to eliminate the negative side-lobes this produces, we apodized the interferograms using the extended Norton-Beer function 1.5 (Naylor \& Tahic 2007). This results in line profiles which are close to Gaussian, with a full width at half-maximum $(F W H M)$ of $0.070 \mathrm{~cm}^{-1}(2.1 \mathrm{GHz})$. We then measured line intensities using the ELF Gaussian fitting routine in DIPSO (Howarth et al. 2004). We detected 203 lines in AFGL 2688,

\footnotetext{
$\overline{{ }^{1} \text { http://www.univie.ac } . a t / s p a c e / M E S S / ~}$
}

214 in AFGL 618 and 164 in NGC 7027. We present plots of the continuum-subtracted spectra in Fig. 1.

\section{Line measurements}

Most strong lines in our spectra are readily identified, although a number of lines remain unidentified at this stage. Table 1 , available online, contains measured line intensities and identifications for each object spectrum; Tables 2-4 list the strongest unidentified lines. The quoted uncertainties are from the line fitting only; the absolute uncertainties on the flux calibration are estimated to be $10-20 \%$ in the $S S W$ band and $\sim 30 \%$ in the $S L W$ band.

CO: All three spectra are dominated by the rotational lines of ${ }^{12} \mathrm{CO}$. We plot rotational diagrams using the method described by Justtanont et al. (2000). Figure 2 shows plots for ${ }^{12} \mathrm{CO}$ and ${ }^{13} \mathrm{CO}$ for our targets, using all the observed lines, from $J=4-3$ to $J=13-12$ for ${ }^{12} \mathrm{CO}$, and $J=5-4$ to $J=14-13$ for ${ }^{13} \mathrm{CO}$. The CO $J=10-9$ line at $38.426 \mathrm{~cm}^{-1}$ is blended with $\mathrm{HCN} J=13-$ 12 at $38.408 \mathrm{~cm}^{-1}$, and we estimate the relative contribution of the two species by linearly interpolating between the fluxes of the two neighbouring lines. The sum of these estimated fluxes is within 3 per cent of the measured flux of the blend. 
R. Wesson et al.: Herschel-SPIRE FTS spectroscopy of the carbon-rich objects AFGL 2688, AFGL 618, and NGC 7027
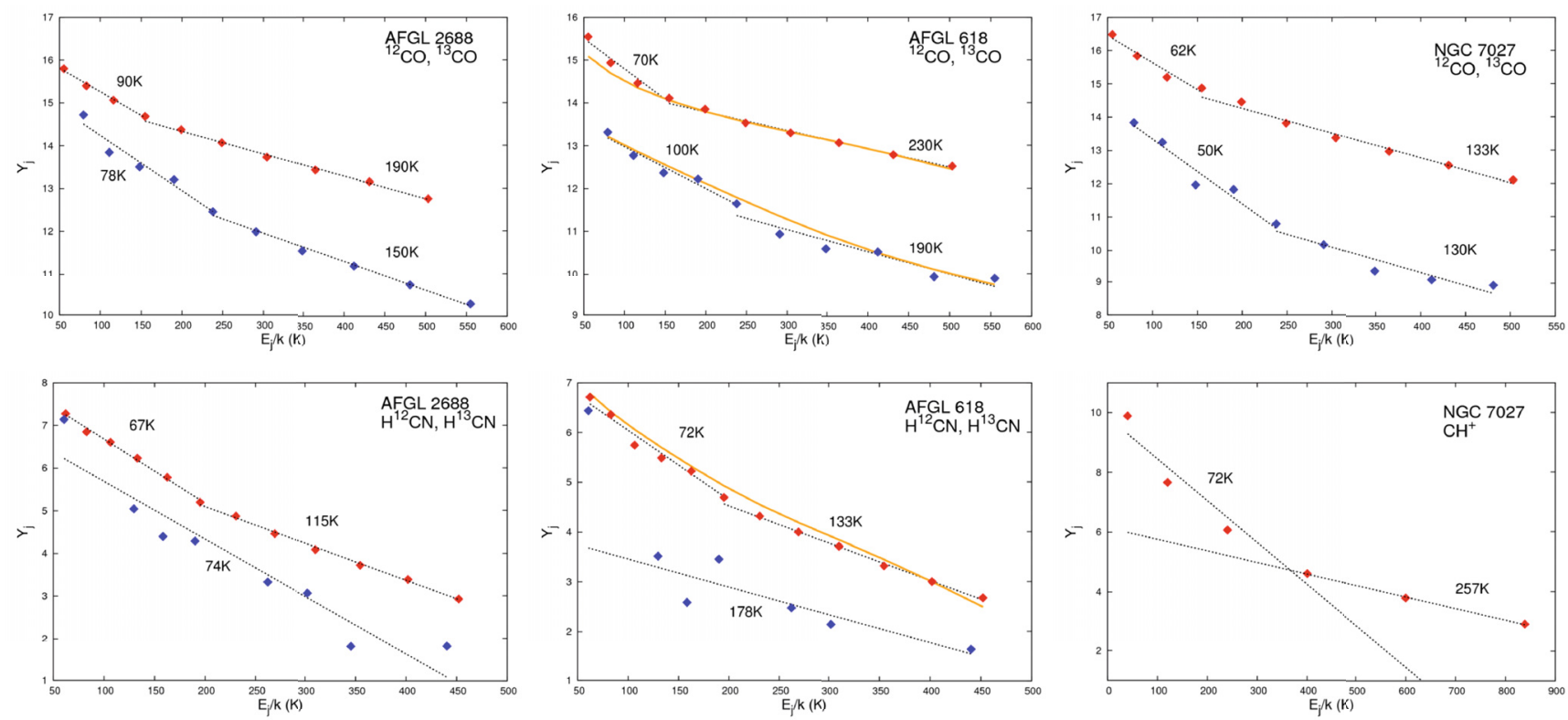

Fig. 2. Rotation diagrams based on fluxes measured in our SPIRE FTS spectra. $Y_{j}=\ln \left(4 \pi F_{j i} / A_{j i} h v_{j i} g_{j}\right)$, where $F_{j i}$ is the line flux in Wm ${ }^{-2}, A_{j i}$ is the Einstein coefficient for spontaneous emission, $v_{j i}$ is the frequency of the transition and $g_{j}$ is the statistical weight of the upper level $j$. The object and species are labelled in each panel. Red points indicate species containing ${ }^{12} \mathrm{C}$, and blue points indicate ${ }^{13} \mathrm{C}$. The temperatures derived from the slopes of the diagrams are indicated. The $\mathrm{CO}, \mathrm{H}^{12} \mathrm{CN}$ and $\mathrm{CH}^{+}$data points clearly steepen at lower $E_{j}$ and we fit these data with two components. $\mathrm{H}^{13} \mathrm{CN}$ data points are fitted with a single line. The orange lines on the diagrams for AFGL 618 represent the predictions of our LVG models, described in Sect. 4.

We derive rotational temperatures by fitting a line to the observed points, with the slope of the line being equal to $1 / T_{\text {rot }}$. The data clearly cannot be fitted by a single temperature, and so for ${ }^{12} \mathrm{CO}$ we fit the data for $J_{\text {up }} \leq 7$ and $J_{\text {up }} \geq 8$ separately, while for ${ }^{13} \mathrm{CO}$ we fit the data for $J_{\text {up }} \leq 9$ and $J_{\text {up }} \geq 10$ separately. The temperatures we derive lie between 60 and $230 \mathrm{~K}$, and are shown on the plots in Fig. 2. The uncertainties on the rotational temperatures are typically $10-20 \mathrm{~K}$.

Justtanont et al. (2000) find considerably higher temperatures following this approach for lines up to $J=37-36$ observed in ISO-LWS spectra; plotting the new Herschel data together with the ISO data clearly shows that the $\mathrm{CO}$ lines cannot be described by a uniform rotational temperature, with the higher- $J$ lines requiring higher temperatures. This indicates that the low- $J$ lines are tracing the extended AGB envelope, while the higher- $J$ lines are tracing hotter gas from the inner layers of the outflow. Models produced by Herpin \& Cernicharo (2000) and Herpin et al. (2002) use several spatial components at different temperatures to reproduce the observed emission from these objects.

HCN: We also plot rotation diagrams for $\mathrm{H}^{12} \mathrm{CN}$ and $\mathrm{H}^{13} \mathrm{CN}$ in AFGL 2688 and AFGL 618, where these species are well detected (Fig. 2). We derive rotation temperatures from fits to the observed points, using the interpolated flux described above for the $J=13-12$ transition of $\mathrm{H}^{12} \mathrm{CN}$, which is blended with the ${ }^{12} \mathrm{CO} J=10-9$ line. The temperatures we derive are comparable to those derived from the ${ }^{13} \mathrm{CO}$ lines, suggesting that these species trace similar regions of the circumstellar environments.

Rotational lines of vibrationally-excited HCN lines can be prominent in the AGB phase of carbon-rich stars (Cernicharo et al. 1996), and we detect them in AFGL 2688 and AFGL 618. At the resolution of the SPIRE spectrometer, the $l=-1$ component of the $v_{2}=1$ level is blended with the ground state HCN lines, while the $l=+1$ component lines lie within one resolution element of those of $\mathrm{HCO}^{+}$; ionised molecular species have very low abundances in AFGL 2688 and so we attribute lines at the frequencies of HCN $v_{2}=1$ to this species alone. In AFGL 618, if the blend is not resolved we attribute the lines to both $\mathrm{HCO}^{+}$and $\mathrm{HCN} v_{2}=1$.

$\mathbf{H}_{2}$ O: Herpin \& Cernicharo (2000) detected lines due to ortho- $\mathrm{H}_{2} \mathrm{O}$ in AFGL 618 in ISO spectra. We now also detect para- $\mathrm{H}_{2} \mathrm{O}$ in this object, and we also detect water lines from AFGL 2688 for the first time. We also detect seven lowlying water lines from NGC 7027. The origin of oxygen-bearing molecules in carbon-rich objects is not well understood, given the expectation that all oxygen molecules will be locked up in $\mathrm{CO}$ with none left over to form oxygen-bearing molecules. They may trace much older regions of the outflow from the star, from a time when its surface composition was oxygenrich. Cernicharo et al. (2004) showed that photodissociation of $\mathrm{CO}$ by the stellar UV field followed by reactions with $\mathrm{H}_{2}$ can account for the observed abundance of $\mathrm{H}_{2} \mathrm{O}$ in AFGL 618; AFGL 2688's much cooler central star makes a similar origin unlikely. Alternative production mechanisms include the vaporization of icy bodies (Melnick et al. 2001), Fischer-Tropsch catalysis on grain surfaces (Willacy 2004) and radiative association between atomic oxygen and molecular hydrogen during the AGB phase (Agúndez \& Cernicharo 2006). Another possibility that should be explored is how deep interstellar UV photons can penetrate into the envelope. The circumstellar envelope of AFGL 2688 is formed by a series of concentric shells rather than a continuous envelope (Sahai et al. 1998), and if interstellar UV photons can reach its warm inner regions, then a very rich chemistry could be triggered by the photodissociation of $\mathrm{CO}$ and other species. In the case of NGC 7027, water lines were tentatively identified in ISO-LWS spectra by Cernicharo et al. (1997) and Liu et al. (1997). Yan et al. (1999) and Agúndez et al. (2010) showed that $\mathrm{H}_{2} \mathrm{O}$ could form in NGC 7027's warm, dense PDR through reactions involving vibrationally-excited $\mathrm{H}_{2}$.

$\mathbf{C H}^{+}$: Lines due to ionised species are absent in the spectrum of AFGL 2688, weak in AFGL 618 and strong in NGC 7027. The $\mathrm{CH}^{+}$line at $27.855 \mathrm{~cm}^{-1}$ is very bright in NGC 7027, and 
we combine our observation with LWS observations of higher- $J$ $\mathrm{CH}^{+}$lines from Cernicharo et al. (1997) to produce the rotation diagram in Fig. 2. The data cannot be fitted by a single temperature, and we derive $T_{\text {rot }}=72 \pm 12 \mathrm{~K}$ for $E_{j}<400 \mathrm{~K}$, and $T_{\text {rot }}=257 \pm 8 \mathrm{~K}$ for $E_{j}>400 \mathrm{~K}$. These temperatures are far lower than the $\sim 4300 \mathrm{~K}$ activation energy of the reaction $\mathrm{C}^{+}+\mathrm{H}_{2} \rightarrow \mathrm{CH}^{+}+\mathrm{H}$. However, under the simplifying assumptions of low optical depth and LTE, the derived rotational temperature is a lower limit to the kinetic temperature. Agúndez et al. (2010) find that $\mathrm{CH}^{+}$in NGC 7027 is formed both via this reaction in warm gas $\left(T_{\mathrm{k}}>400-500 \mathrm{~K}\right)$ and via the reaction of vibrationally excited $\mathrm{H}_{2}$ with $\mathrm{C}^{+}$.

\section{LVG models for AFGL 618}

To further investigate the nature of AFGL 618, we constructed a large velocity gradient (LVG) model, based initially on that of Herpin \& Cernicharo (2000), in which the object is modelled as a torus surrounded by a high-velocity wind (HVW) region, with the ejecta from the earlier AGB phase further out. Material in the torus is at temperatures from $250-1000 \mathrm{~K}$; the HVW region consists mostly of $200 \mathrm{~K}$ material with a small amount of hotter gas at $1000 \mathrm{~K}$, and the AGB remnant has a temperature of $100 \mathrm{~K}$. The $\mathrm{CO}$ rotation temperatures derived by Justtanont et al. (2000) from ISO data, and in this paper from Herschel data, are consistent with the model temperatures, with ISO sampling the warmer material and Herschel being sensitive to the cooler material further from the central star. We used the model to predict ${ }^{12} \mathrm{CO},{ }^{13} \mathrm{CO}, \mathrm{H}^{12} \mathrm{CN}$ and $\mathrm{H}^{13} \mathrm{CN}$ fluxes.

Matching our observed ${ }^{12} \mathrm{CO}$ and ${ }^{13} \mathrm{CO}$ fluxes was possible with almost exactly the same model parameters for $\mathrm{CO}$ as given in Table 1 of Herpin \& Cernicharo; the only change was to reduce the ${ }^{13} \mathrm{CO}$ column density in the $\mathrm{AGB}$ ejecta from $3.5 \times 10^{17} \mathrm{~cm}^{-2}$ to $2.5 \times 10^{17} \mathrm{~cm}^{-2}$, reducing the total ${ }^{13} \mathrm{CO}$ column density by $7 \%$. This is well within the estimated model uncertainties of $20-30 \%$. A good match to the observed $\mathrm{H}^{12} \mathrm{CN}$ intensities required a total column density of $\mathrm{HCN}$ a factor of 5 lower than the value found by Herpin \& Cernicharo (2000). This reflects the different regimes to which ISO and Herschel are sensitive, with Herschel providing information about much cooler regions than could be observed with ISO. The match to the $\mathrm{H}^{13} \mathrm{CN}$ intensities is poor; these lines are quite weak in AFGL 618 and may be blended. We plot the predictions of the model for ${ }^{12} \mathrm{CO},{ }^{13} \mathrm{CO}$ and $\mathrm{H}^{12} \mathrm{CN}$ on the rotation diagrams in Fig. 2.

The ${ }^{12} \mathrm{C} /{ }^{13} \mathrm{C}$ ratio is a tracer of the history of nuclear processing inside stars. ${ }^{13} \mathrm{C}$ is an intermediate product in the $\mathrm{CNO}$ cycle which is the main fusion reaction converting hydrogen into helium in stars with masses $>1.2 M_{\odot}$, and lower ${ }^{12} \mathrm{C} /{ }^{13} \mathrm{C}$ ratios result from $\mathrm{CNO}$ cycle material being brought to the surface. Milam et al. (2009) find that carbon-rich stars have ${ }^{12} \mathrm{CO} /{ }^{13} \mathrm{CO}$ ratios generally in the range $25-90$. To match the observed CO line fluxes in AFGL 618 requires a ${ }^{12} \mathrm{C} /{ }^{13} \mathrm{C}$ ratio of 21 in our model, implying substantial ${ }^{13} \mathrm{C}$ enrichment. Pardo \& Cernicharo (2007) find a ${ }^{12} \mathrm{C} /{ }^{13} \mathrm{C}$ ratio of $\sim 15$ in regions closest to the central star and $\sim 40$ in regions further out, possibly indicating a late ejection of ${ }^{13} \mathrm{C}$-rich material from the star.

\section{Conclusions}

The unprecedented wavelength coverage and excellent sensitivity of the SPIRE spectrometer have allowed us to see the incredibly rich far-infrared and submm spectra of three carbon-rich evolved stars for the first time. We have detected 150-200 emission lines in the SPIRE FTS spectra of AFGL 2688, AFGL 618 and NGC 7027, and presented preliminary investigations of the physical conditions in these objects based on the new data. We find water in both ortho and para forms in all three objects, and observe strong ionised lines in the more evolved NGC 7027, including the $J=1-0$ line of $\mathrm{CH}^{+}$. This line, never previously detected in an astronomical source, is also detected in Herschel spectra of H II region PDRs (Naylor et al. 2010). LVG models of AFGL 618 are in excellent agreement with those based on earlier ISO data. These observations show that the SPIRE spectrometer on board Herschel can provide significant new insights into the temperatures structure and chemical content of the outflows from evolved stars.

Acknowledgements. SPIRE has been developed by a consortium of institutes led by Cardiff Univ, (UK) and including Univ. Lethbridge (Canada); NAOC (China); CEA, LAM (France); IFSI, Univ. Padua (Italy); IAC (Spain); Stockholm Observatory (Sweden); Imperial College London, RAL, UCL-MSSL, UKATC, Univ. Sussex (UK); and Caltech, JPL, NHSC, Univ. Colorado (USA). This development has been supported by national funding agencies: CSA (Canada); NAOC (China); CEA, CNES, CNRS (France); ASI (Italy); MCINN (Spain); SNSB (Sweden); STFC (UK); and NASA (USA). We thank Spanish MICINN for funding support through grants AYA2006-14876, AYA2009-07304 and the CONSOLIDER program "ASTROMOL" CSD2009-00038. M.A.T.G. and K.M.E. acknowledge support from the Belgian Federal Science Policy Office via the PRODEX Programme of ESA. RW thanks Estelle Bayet for helpful discussions.

\section{References}

Agúndez, M., \& Cernicharo, J. 2006, ApJ, 650, 374

Agúndez, M., Goicoechea, J. R., Cernicharo, J., et al. 2010, ApJ, 713, 662 Bujarrabal, V., Gomez-Gonzalez, J., Bachiller, R., \& Martin-Pintado, J. 1988, A\&A, 204, 242

Cernicharo, J., Barlow, M. J., González-Alfonso, E., et al. 1996, A\&A, 315, 201 Cernicharo, J., Liu, X.-W., González-Alfonso, E., et al. 1997, ApJ, 483, L65 Cernicharo, J. 2004, ApJ, 608, 41

Cox, P., González-Alfonso, E., Barlow, M. J., et al. 1996, A\&A, 315, 265 Frew, D. J., \& Parker, Q. A. 2010, PASA, 27, 129

Griffin, M. J., Abergel, A., Abreu, A., et al. 2010, A\&A, 518, L3 Herpin, F., \& Cernicharo, J. 2000, ApJ, 530, 129

Herpin, F., Goicoechea, J. R., Pardo, J. R., \& Cernicharo, J. 2002, ApJ, 577, 961 Howarth, I. D., Murray, J., \& Mills, D. 1994, Starlink User Note, No. 50.15 Justtanont, K., Barlow, M. J., Tielens A. G. G. M., et al. 2000, A\&A, 360, 1117 Kwok, S., \& Feldman, P. A. 1981, ApJ, 247, L67

Kwok, S., \& Bignell, R. C. 1984, ApJ, 276, 544

Liu, X.-W., Barlow, M. J., Nguyen-Q-Rieu, et al. 1996, A\&A, 315, 257

Liu, X.-W., Barlow, M. J., Dalgarno, A., et al. 1997, MNRAS, 290, 71 Masson, C. R. 1989, ApJ, 336, 294

Matsuura, M., Barlow, M. J., Zijlstra, A. A., et al. 2009, MNRAS, 396, 918

Melnick, G., et al. 2001, Nature, 412, 160

Milam, S. N., Woolf, N. J., \& Ziurys, L. M. 2009, ApJ, 690, 837

Naylor, D. A., \& Tahic, M. K. 2007, J. Opt. Soc. Am. A, 24, 3644

Naylor, D. A., Dartois, E., Habart, E., et al. 2010, A\&A, 518, L117

Pardo, J. R., \& Cernicharo, J. 2007, ApJ, 654, 978

Pardo, J. R., Cernicharo, J., Goicoechea, J. R., \& Phillips, T. G. 2004, ApJ, 615, 495

Pardo, J. R., Cernicharo, J., \& Goicoechea, J. R. 2005, ApJ, 628, 275

Pardo, J. R., Cernicharo, J., Goicoechea, J. R., Guélin, M., \& Asensio Ramos, A. 2007, ApJ, 661, 250

Pilbratt, G. L., Riedinger, J. R., Passvogel, T., et al. 2010, A\&A, 518, L1

Sahai, R., Trauger, J. T., Watson, A. M., et al. 1998, ApJ, 493, 301

Sánchez Contreras, C., Bujarrabal, V., Castro-Carrizo, A., Alcolea, J., \& Sargent, A. 2004, ApJ, 617, 1142

Swinyard, B. M., Ade, P., Baluteau, J.-P., et al. 2010, A\&A, 518, L4

Szczerba, R., Siódmiak, N., Stasińska, G., \& Borkowski, J. 2007, A\&A, 469, 799

Ueta, T., Murakawa, K., \& Meixner, M. 2006, ApJ, 641, 1113

Willacy 2004, ApJ, 600, 87

Wynn-Williams, C. G. 1977, MNRAS, 181, 61

Yan, M., Federman, S. R., Dalgarno, A., \& Bjorkman, J. E. 1999, ApJ, 515, 640

Pages 5 to 8 are available in the electronic edition of the journal at http://www . aanda.org 
1 Department of Physics and Astronomy, University College London, Gower Street, London WC1E 6BT, UK

e-mail: rwesson@star.ucl.ac.uk

2 Astrophysics Dept, CAB (INTA-CSIC), Crta Ajalvir km4, 28805 Torrejon de Ardoz, Madrid, Spain

${ }^{3}$ Mullard Space Science Laboratory, University College London, Holmbury St. Mary, Dorking, Surrey RH5 6NT, UK

${ }^{4}$ Instituut voor Sterrenkunde, Katholieke Universiteit Leuven, Celestijnenlaan 200 D, 3001 Leuven, Belgium

5 Universiteit van Amsterdam, Sterrenkundig Instituut "Anton Pannekoek", Science Park 904, 1098 XH Amsterdam, The Netherlands

${ }^{6}$ Royal Observatory of Belgium, Ringlaan 3, 1180 Brussels, Belgium

7 Space Science and Technology Department, Rutherford Appleton Laboratory, Oxfordshire, OX11 0QX, UK
8 Institute for Space Imaging Science, University of Lethbridge, 4401 University Drive, Lethbridge, Alberta, T1K 3M4, Canada

9 LUTH, Observatoire de Paris-Meudon, 5 Place Jules Janssen, 92190 Meudon, France

10 Radio Astronomy Laboratory, University of California at Berkeley, CA 94720, USA

11 School of Physics and Astronomy, Cardiff University, The Parade, Cardiff, Wales CF24 3AA, UK

12 Blue Sky Spectroscopy, 9/740 4 Ave S, Lethbridge, Alberta T1J 0N9, Canada

13 UK Astronomy Technology Centre, Royal Observatory Edinburgh, Blackford Hill, Edinburgh EH9 3HJ, UK

14 Dept of Astronomy, Stockholm University, AlbaNova University Center, Roslagstulsbacken 21, 10691 Stockholm, Sweden

15 Dept. of Physics and Astronomy, University of Denver, Mail Stop 6900, Denver, CO 80208, USA 
A\&A 518, L144 (2010)

Table 1. Line intensities and identifications in each spectrum. Rows beginning with a "+" indicate that the line is blended with that on the row above. The quantum numbers given in the "transition" column are $J$ except where stated. $\mathrm{H}_{2} \mathrm{O}$ transitions are given in the format $J^{\prime}{ }_{k_{a}^{\prime}} k_{c}^{\prime}-J_{k_{a} k_{c}}$.

\begin{tabular}{|c|c|c|c|c|c|c|c|c|c|}
\hline \multirow[b]{2}{*}{$\bar{v}_{0}\left(\mathrm{~cm}^{-1}\right)$} & \multirow[b]{2}{*}{$\lambda_{0}(\mu \mathrm{m})$} & \multirow[b]{2}{*}{ Species } & \multirow[b]{2}{*}{ Transition } & \multicolumn{2}{|c|}{ AFGL 2688} & \multicolumn{2}{|c|}{ AFGL 618} & \multicolumn{2}{|c|}{ NGC 7027} \\
\hline & & & & $\bar{v}_{\mathrm{obs}}\left(\mathrm{cm}^{-1}\right)$ & $\mathrm{F}\left(\times 10^{-18} \mathrm{Wm}^{-2}\right)$ & $\bar{v}_{\text {obs }}\left(\mathrm{cm}^{-1}\right)$ & $\mathrm{F}\left(\times 10^{-18} \mathrm{Wm}^{-2}\right)$ & $\bar{v}_{\text {obs }}\left(\mathrm{cm}^{-1}\right)$ & $\mathrm{F}\left(\times 10^{-18} \mathrm{Wm}^{-2}\right)$ \\
\hline 15.119 & 661.40 & HNC & $5-4$ & & & 15.111 & $205.8 \pm 6.3$ & & \\
\hline 15.239 & 656.23 & ${ }^{12} \mathrm{CO}$ & $v=1 ; 4-3$ & 15.248 & $63.0 \pm 8.1$ & & & & \\
\hline 15.379 & 650.25 & ${ }^{12} \mathrm{CO}$ & $4-3$ & 15.372 & $281.4 \pm 8.7$ & 15.369 & $217.8 \pm 12.9$ & 15.371 & $556.5 \pm 6.0$ \\
\hline 15.707 & 636.65 & $\mathrm{p}-\mathrm{H}_{2} \mathrm{O}$ & $6_{42}-5_{51}$ & & & 15.705 & $20.1 \pm 18.3$ & & \\
\hline 16.294 & 613.71 & $\mathrm{p}-\mathrm{H}_{2} \mathrm{O}$ & $624-7_{17}$ & & & 16.275 & $56.4 \pm 13.8$ & 16.282 & $129.9 \pm 21.9$ \\
\hline 16.417 & 609.14 & {$[\mathrm{C} \mathrm{I}]$} & $1-0$ & & & & & 16.397 & $97.2 \pm 21.6$ \\
\hline 17.278 & 578.78 & $\mathrm{H}^{13} \mathrm{CN}$ & $6-5$ & 17.273 & $256.8 \pm 2.7$ & 17.293 & $127.5 \pm 9.0$ & 17.259 & $126.3 \pm 28.5$ \\
\hline 17.428 & 573.80 & $\mathrm{HN}^{13} \mathrm{C}$ & $6-5$ & 17.411 & $91.5 \pm 8.7$ & & & 17.420 & $60.0 \pm 13.8$ \\
\hline 17.478 & 572.16 & $\mathrm{CCH}$ & $N=6-5$ & & & 17.472 & $165.3 \pm 11.7$ & & \\
\hline 17.736 & 563.82 & $\mathrm{HCN}$ & $6-5$ & 17.733 & $325.8 \pm 6.6$ & 17.742 & $185.7 \pm 4.2$ & 17.748 & $31.8 \pm 11.4$ \\
\hline 17.848 & 560.30 & $\mathrm{HCO}^{+}$ & $6-5$ & & & 17.864 & $126.3 \pm 3.6$ & & \\
\hline 18.377 & 544.16 & ${ }^{13} \mathrm{CO}$ & $5-4$ & 18.375 & $243.6 \pm 7.8$ & 18.364 & $59.7 \pm 9.0$ & 18.377 & $100.5 \pm 3.9$ \\
\hline 18.577 & 538.29 & $\mathrm{o}-\mathrm{H}_{2} \mathrm{O}$ & $1_{10}-1_{01}$ & 18.571 & $164.4 \pm 3.9$ & 18.583 & $68.1 \pm 4.5$ & 18.574 & $154.5 \pm 14.7$ \\
\hline 19.222 & 520.23 & ${ }^{12} \mathrm{CO}$ & $5-4$ & 19.222 & $568.2 \pm 4.2$ & 19.223 & $358.2 \pm 1.5$ & 19.219 & $882.9 \pm 4.8$ \\
\hline 20.390 & 490.45 & $\mathrm{CCH}$ & $N=7-6$ & 20.384 & $39.6 \pm 2.4$ & 20.390 & $40.8 \pm 5.1$ & 20.374 & $42.3 \pm 4.8$ \\
\hline 20.691 & 483.30 & $\mathrm{HCN}$ & $7-6$ & 20.687 & $456.9 \pm 3.3$ & 20.687 & $279.9 \pm 6.6$ & 20.674 & $43.5 \pm 2.7$ \\
\hline 20.793 & 480.93 & $\mathrm{HCN}$ & $v_{2}=1 ; 7-6 f$ & 20.787 & $111.6 \pm 3.3$ & & & & \\
\hline 20.821 & 480.28 & $\mathrm{HCO}^{+}$ & $7-6$ & & & 20.818 & $150.6 \pm 6.9$ & 20.818 & $157.8 \pm 3.3$ \\
\hline 20.878 & 478.96 & $\mathrm{HCl}$ & $1-0$ & 20.859 & $100.5 \pm 3.3$ & & & & \\
\hline 21.165 & 472.48 & $\mathrm{HNC}$ & $7-6$ & & & 21.176 & $89.4 \pm 4.5$ & & \\
\hline 21.949 & 455.61 & $\mathrm{p}-\mathrm{H}_{2} \mathrm{O}$ & $v_{2}=1 ; 1_{10}-1_{01}$ & 21.946 & $70.5 \pm 7.8$ & & & & \\
\hline 22.051 & 453.50 & ${ }^{13} \mathrm{CO}$ & $6-5$ & 22.044 & $249.3 \pm 5.1$ & 22.054 & $85.5 \pm 3.0$ & 22.044 & $136.8 \pm 4.8$ \\
\hline 22.483 & 444.79 & $\mathrm{CN}$ & $N=6-5 ; v=1$ & 22.489 & $11.7 \pm 2.7$ & & & & \\
\hline 22.683 & 440.86 & $\mathrm{CN}$ & $N=6-5 ; v=0$ & 22.681 & $73.5 \pm 5.7$ & & & & \\
\hline 22.855 & 437.54 & ${ }^{12} \mathrm{CO}$ & $v=1 ; 6-5$ & 22.849 & $50.1 \pm 3.3$ & 22.872 & $45.6 \pm 38.1$ & & \\
\hline 23.065 & 433.56 & ${ }^{12} \mathrm{CO}$ & $6-5$ & 23.060 & $1002.0 \pm 3.6$ & 23.064 & $547.8 \pm 4.8$ & 23.058 & $1144.0 \pm 5.1$ \\
\hline 23.303 & 429.13 & $\mathrm{CCH}$ & $N=8-7$ & 23.309 & $58.5 \pm 3.9$ & 23.309 & $76.2 \pm 10.5$ & 23.306 & $33.9 \pm 3.6$ \\
\hline 23.646 & 422.91 & $\mathrm{HCN}$ & $8-7$ & 23.643 & $693.9 \pm 4.5$ & 23.643 & $293.4 \pm 5.1$ & 23.636 & $40.8 \pm 3.6$ \\
\hline 23.762 & 420.84 & $\mathrm{HCN}$ & $v_{2}=1 ; 8-7 \mathrm{f}$ & 23.761 & $101.4 \pm 3.6$ & & & & \\
\hline 23.795 & 420.26 & $\mathrm{HCO}^{+}$ & $8-7$ & & & 23.789 & $118.2 \pm 6.0$ & 23.795 & $40.8 \pm 4.2$ \\
\hline 24.187 & 413.45 & $\mathrm{HNC}$ & $8-7$ & 24.195 & $30.6 \pm 9.3$ & 24.191 & $110.7 \pm 1.8$ & & \\
\hline 25.085 & 398.64 & $\mathrm{o}-\mathrm{H}_{2} \mathrm{O}$ & $2_{11}-2_{02}$ & & & 25.113 & $228.3 \pm 4.5$ & & \\
\hline 25.492 & 392.28 & $\mathrm{H} \mathrm{I}$ & $\mathrm{H} 20 \alpha$ & & & 25.507 & $50.1 \pm 3.9$ & 25.493 & $6.6 \pm 3.3$ \\
\hline 25.724 & 388.74 & ${ }^{13} \mathrm{CO}$ & $7-6$ & 25.720 & $380.1 \pm 7.5$ & 25.719 & $121.8 \pm 3.0$ & 25.720 & $81.6 \pm 4.5$ \\
\hline 25.912 & 385.92 & $\mathrm{H}^{13} \mathrm{CN}$ & $9-8$ & 25.914 & $235.2 \pm 6.9$ & 25.915 & $51.0 \pm 1.5$ & & \\
\hline 26.171 & 382.10 & $\mathrm{CN}$ & $N=7-6 ; v=1$ & & & & & 26.167 & $28.5 \pm 4.2$ \\
\hline 26.226 & 381.30 & $\mathrm{CCH}$ & $N=9-8$ & 26.225 & $148.2 \pm 6.9$ & 26.233 & $147.0 \pm 3.0$ & 26.230 & $42.9 \pm 5.1$ \\
\hline 26.470 & 377.78 & $\mathrm{CN}$ & $N=7-6 ; v=0$ & 26.468 & $56.4 \pm 20.1$ & & & & \\
\hline 26.600 & 375.95 & $\mathrm{HCN}$ & $9-8$ & 26.596 & $855.0 \pm 9.6$ & 26.599 & $406.2 \pm 6.3$ & & \\
\hline 26.731 & 374.10 & $\mathrm{HCN}$ & $v_{2}=1 ; 9-8 \mathrm{f}$ & 26.732 & $98.4 \pm 8.7$ & 26.756 & $166.2 \pm 6.6$ & & \\
\hline 26.767 & 373.59 & $\mathrm{HCO}^{+}$ & $9-8$ & & & & & & \\
\hline $\begin{array}{l}26.907 \\
26.997\end{array}$ & 371.65 & ${ }^{12} \mathrm{CO}$ & $7-6$ & 26.905 & $1465.0 \pm 9.3$ & 26.907 & $825.9 \pm 6.3$ & $\begin{array}{l}26.900 \\
26.997\end{array}$ & $\begin{array}{l}1768.0 \pm 13.8 \\
113.4 \pm 11.1\end{array}$ \\
\hline $\begin{array}{l}26.997 \\
27.208\end{array}$ & $\begin{array}{l}370.41 \\
367.54\end{array}$ & $\begin{array}{l}{[\mathrm{C} \text { I] }} \\
\mathrm{HNC}\end{array}$ & $\begin{array}{l}2-1 \\
9-8\end{array}$ & & & 27.211 & $113.7 \pm 2.1$ & & \\
\hline 27.855 & 359.00 & $\mathrm{CH}^{+}$ & $1-0$ & & & 27.847 & $65.1 \pm 3.0$ & 27.851 & $466.5 \pm 4.5$ \\
\hline 28.685 & 348.61 & $\mathrm{o}-\mathrm{H}_{2} \mathrm{O}$ & $v_{2}=1 ; 2_{11}-2_{02}$ & 28.677 & $60.9 \pm 2.1$ & 28.672 & $32.7 \pm 8.4$ & & \\
\hline 28.789 & 347.36 & $\mathrm{H}^{13} \mathrm{CN}$ & $10-9$ & 28.781 & $207.3 \pm 2.7$ & 28.775 & $33.9 \pm 7.2$ & & \\
\hline 29.121 & 343.39 & $\mathrm{CCH}$ & $N=10-9$ & 29.129 & $146.1 \pm 7.8$ & 29.139 & $91.8 \pm 8.7$ & 29.119 & $57.6 \pm 10.2$ \\
\hline 29.396 & 340.18 & ${ }^{13} \mathrm{CO}$ & $8-7$ & 29.391 & $543.9 \pm 5.4$ & 29.389 & $202.8 \pm 8.7$ & 29.386 & $136.8 \pm 12.6$ \\
\hline 29.553 & 338.38 & $\mathrm{HCN}$ & $10-9$ & 29.550 & $916.8 \pm 5.7$ & 29.556 & $524.1 \pm 9.3$ & & \\
\hline 29.622 & 337.59 & $\mathrm{H} \mathrm{I}$ & $\mathrm{H} 19 \alpha$ & & & & & 29.614 & $10.2 \pm 6.0$ \\
\hline 29.698 & 336.72 & $\mathrm{HCN}$ & $v_{2}=1 ; 10-9 \mathrm{f}$ & 29.695 & $172.8 \pm 6.9$ & 29715 & $1896+81$ & & \\
\hline 29.739 & 336.26 & $\mathrm{HCO}^{+}$ & $10-9$ & & & 29.115 & $189.0 \pm 8.1$ & 29.735 & $7.8 \pm 5.7$ \\
\hline 29.970 & 333.67 & $\mathrm{CN}$ & $N=8-7 ; v=1$ & 29.974 & $117.3 \pm 13.2$ & & & & \\
\hline 30.228 & 330.82 & $\mathrm{p}-\mathrm{H}_{2} \mathrm{O}$ & $9_{28}-8_{35}$ & 30.244 & $167.7 \pm 13.5$ & & & & \\
\hline+30.229 & 330.81 & $\mathrm{HNC}$ & $10-9$ & & & & & & \\
\hline+30.241 & 330.68 & $\mathrm{CN}$ & $N=8-7 ; v=0$ & & & & & & \\
\hline 30.468 & 328.21 & ${ }^{12} \mathrm{CO}$ & $v=1 ; 8-7$ & & & 30.472 & $21.6 \pm 8.4$ & & \\
\hline 30.560 & 327.22 & $\mathrm{p}-\mathrm{H}_{2} \mathrm{O}$ & $4_{22}-3_{31}$ & & & 30.573 & $26.4 \pm 8.7$ & & \\
\hline 30.748 & 325.23 & ${ }^{12} \mathrm{CO}$ & $8-7$ & 30.748 & $2073.0 \pm 16.5$ & 30.748 & $1229.0 \pm 10.2$ & 30.740 & $2249.0 \pm 11.7$ \\
\hline 31.665 & 315.80 & $\mathrm{H}^{13} \mathrm{CN}$ & $11-10$ & 31.653 & $297.3 \pm 24.0$ & 31.651 & $129.3 \pm 19.5$ & & \\
\hline 32.030 & 312.21 & $\mathrm{CCH}$ & $N=11-10$ & & & 32.023 & $69.9 \pm 8.1$ & & \\
\hline 32.366 & 308.96 & $\mathrm{p}-\mathrm{H}_{2} \mathrm{O}$ & $5_{24}-4_{31}$ & & & 32.368 & $81.0 \pm 3.6$ & & \\
\hline 32.505 & 307.64 & $\mathrm{HCN}$ & $11-10$ & 32.501 & $818.7 \pm 6.3$ & 32.501 & $554.1 \pm 3.6$ & & \\
\hline 32.665 & 306.13 & $\mathrm{HCN}$ & $v_{2}=1 ; 11-10 \mathrm{f}$ & 32.665 & $150.3 \pm 7.8$ & 32.703 & $129.3 \pm 10.5$ & & \\
\hline 32.711 & 305.71 & $\mathrm{HCO}^{+}$ & $11-10$ & & & 32.103 & $129.3 \pm 10.5$ & 32.702 & $38.7 \pm 2.7$ \\
\hline 32.954 & 303.46 & $\mathrm{p}-\mathrm{H}_{2} \mathrm{O}$ & $2_{02}-1_{11}$ & 32.946 & $236.4 \pm 7.2$ & 32.952 & $340.2 \pm 3.6$ & 32.943 & $44.1 \pm 3.3$ \\
\hline 33.067 & 302.41 & ${ }^{13} \mathrm{CO}$ & $9-8$ & 33.066 & $457.2 \pm 7.5$ & 33.066 & $202.5 \pm 3.9$ & 33.060 & $87.0 \pm 3.3$ \\
\hline 33.249 & 300.76 & $\mathrm{HNC}$ & $11-10$ & & & 33.244 & $142.2 \pm 3.6$ & & \\
\hline
\end{tabular}


Table 1. continued.

\begin{tabular}{|c|c|c|c|c|c|c|c|c|c|}
\hline \multirow[b]{2}{*}{$\bar{v}_{0}\left(\mathrm{~cm}^{-1}\right)$} & \multirow[b]{2}{*}{$\lambda_{0}(\mu \mathrm{m})$} & \multirow[b]{2}{*}{ Species } & \multirow[b]{2}{*}{ Transition } & \multicolumn{2}{|c|}{ AFGL 2688} & \multicolumn{2}{|c|}{ AFGL 618} & \multicolumn{2}{|c|}{ NGC 7027} \\
\hline & & & & $\bar{v}_{\mathrm{obs}}\left(\mathrm{cm}^{-1}\right)$ & $\mathrm{F}\left(\times 10^{-18} \mathrm{Wm}^{-2}\right)$ & $\bar{v}_{o b s}\left(\mathrm{~cm}^{-1}\right)$ & $\mathrm{F}\left(\times 10^{-18} \mathrm{Wm}^{-2}\right)$ & $\bar{v}_{\text {obs }}\left(\mathrm{cm}^{-1}\right)$ & $\mathrm{F}\left(\times 10^{-18} \mathrm{Wm}^{-2}\right)$ \\
\hline 33.711 & 296.64 & $\mathrm{CN}$ & $N=9-8 ; v=1$ & & & 33.708 & $33.6 \pm 32.4$ & & \\
\hline 34.026 & 293.90 & $\mathrm{CN}$ & $N=9-8 ; v=0$ & 34.030 & $124.8 \pm 13.8$ & 34.039 & $230.7 \pm 14.4$ & & \\
\hline 34.588 & 289.12 & ${ }^{12} \mathrm{CO}$ & $9-8$ & 34.585 & $2711.0 \pm 17.4$ & 34.586 & $1586.0 \pm 10.2$ & 34.579 & $2114.0 \pm 12.0$ \\
\hline 34.695 & 288.23 & $\mathrm{H} \mathrm{I}$ & $\mathrm{H} 18 \alpha$ & & & & & 34.691 & $28.2 \pm 8.7$ \\
\hline 34.940 & 286.20 & $\mathrm{CCH}$ & $N=12-11$ & & & 34.945 & $75.9 \pm 6.0$ & & \\
\hline 35.457 & 282.03 & $\mathrm{HCN}$ & $12-11$ & 35.456 & $907.8 \pm 8.7$ & 35.457 & $521.7 \pm 4.2$ & & \\
\hline 35.632 & 280.65 & $\mathrm{HCN}$ & $v_{2}=1 ; 12-11 \mathrm{f}$ & 35.639 & $237.6 \pm 14.4$ & 35663 & $1536+48$ & & \\
\hline 35.681 & 280.26 & $\mathrm{HCO}^{+}$ & $12-11$ & & & 35.005 & $155.0 \pm 4.0$ & 35.669 & $31.8 \pm 5.4$ \\
\hline 36.268 & 275.73 & $\mathrm{HNC}$ & $12-11$ & & & 36.266 & $144.0 \pm 6.9$ & & \\
\hline 36.604 & 273.19 & $\mathrm{o}-\mathrm{H}_{2} \mathrm{O}$ & $3_{12}-3_{03}$ & 36.586 & $157.8 \pm 15.6$ & 36.601 & $276.0 \pm 7.5$ & & \\
\hline 36.737 & 272.20 & ${ }^{13} \mathrm{CO}$ & $10-9$ & 36.736 & $479.4 \pm 18.0$ & 36.737 & $166.8 \pm 3.0$ & 6.730 & $77.4 \pm 4.2$ \\
\hline 37.137 & 269.27 & $\mathrm{p}-\mathrm{H}_{2} \mathrm{O}$ & $1_{11}-0_{00}$ & 37.140 & $183.3 \pm 6.9$ & 37.138 & $257.1 \pm 4.2$ & 37.119 & $42.9 \pm 11.7$ \\
\hline 37.416 & 267.27 & $\mathrm{H}^{13} \mathrm{CN}$ & $13-12$ & 37.425 & $258.9 \pm 9.6$ & 37.423 & $110.4 \pm 6.6$ & & \\
\hline 37.451 & 267.01 & $\mathrm{CN}$ & $N=10-9 ; v=1$ & & & & & 37.448 & $21.6 \pm 10.2$ \\
\hline 37.800 & 264.55 & $\mathrm{CN}$ & $N=10-9 ; v=0$ & & & 37.799 & $171.6 \pm 3.3$ & & \\
\hline 37.845 & 264.24 & $\mathrm{CCH}$ & $N=13-12$ & & & & & 37.842 & $35.4 \pm 11.1$ \\
\hline 38.076 & 262.63 & ${ }^{12} \mathrm{CO}$ & $v=1 ; 10-9$ & 38.083 & $67.5 \pm 72.3$ & & & & \\
\hline 38.247 & 261.46 & $\mathrm{o}-\mathrm{H}_{2} \mathrm{O}$ & $7_{25}-8_{18}$ & 38.235 & $16.5 \pm 15.3$ & & & & \\
\hline 38.408 & 260.36 & $\mathrm{HCN}$ & $13-12$ & 38.421 & $4116.0 \pm 23.4$ & 38.424 & $2732.0 \pm 20.1$ & 38.416 & $2275.0 \pm 10.8$ \\
\hline+38.426 & 260.24 & ${ }^{12} \mathrm{CO}$ & $10-9$ & & & & & & \\
\hline 38.597 & 259.09 & $\mathrm{HCN}$ & $v_{2}=1 ; 13-12 \mathrm{f}$ & 38.597 & $140.4 \pm 24.9$ & & & & \\
\hline 38.638 & 258.82 & $\mathrm{o}-\mathrm{H}_{2} \mathrm{O}$ & $6_{34}-5_{41}$ & & & 38.638 & $131.1 \pm 19.2$ & & \\
\hline+38.651 & 258.73 & $\mathrm{HCO}^{+}$ & $13-12$ & & & & & & \\
\hline 38.791 & 257.79 & $\mathrm{o}-\mathrm{H}_{2} \mathrm{O}$ & $3_{21}-3_{12}$ & & & 38.793 & $223.2 \pm 6.3$ & & \\
\hline 38.972 & 256.59 & $\mathrm{o}-\mathrm{H}_{2} \mathrm{O}$ & $8_{54}-7_{61}$ & 38.969 & $250.2 \pm 6.9$ & 38.966 & $205.2 \pm 6.9$ & & \\
\hline 39.286 & 254.55 & $\mathrm{HNC}$ & $13-12$ & 39.293 & $75.0 \pm 5.7$ & 39.279 & $139.5 \pm 5.1$ & & \\
\hline 40.282 & 248.25 & $\mathrm{p}-\mathrm{H}_{2} \mathrm{O}$ & $4_{22}-4_{13}$ & 40.280 & $285.0 \pm 3.9$ & 40.278 & $113.1 \pm 4.2$ & & \\
\hline+40.290 & 248.20 & $\mathrm{H}^{13} \mathrm{CN}$ & $14-13$ & & & & & & \\
\hline 40.406 & 247.49 & ${ }^{13} \mathrm{CO}$ & $11-10$ & 40.401 & $479.7 \pm 4.2$ & 40.402 & $185.7 \pm 4.5$ & 40.392 & $54.9 \pm 4.2$ \\
\hline 40.517 & 246.81 & $\mathrm{p}-\mathrm{H}_{2} \mathrm{O}$ & $v_{2}=1 ; 3_{12}-3_{03}$ & & & & & 40.510 & $5.7 \pm 3.9$ \\
\hline 40.530 & 246.73 & $\mathrm{p}-\mathrm{H}_{2} \mathrm{O}$ & $v_{2}=1 ; 8_{45}-7_{52}$ & 40.525 & $356.1 \pm 3.6$ & 40.524 & $220.2 \pm 3.9$ & & \\
\hline 40.750 & 245.40 & $\mathrm{CCH}$ & $N=14-13$ & & & 40.752 & $64.5 \pm 4.2$ & & \\
\hline 40.988 & 243.97 & $\mathrm{p}-\mathrm{H}_{2} \mathrm{O}$ & $2_{20}-2_{11}$ & & & 40.992 & $102.0 \pm 3.9$ & 40.981 & $31.5 \pm 4.5$ \\
\hline+40.996 & 243.93 & $\mathrm{H} \mathrm{I}$ & $\mathrm{H} 17 \alpha$ & & & & & & \\
\hline 41.191 & 242.77 & $\mathrm{CN}$ & $N=11-10 ; v=1$ & & & 41.201 & $21.3 \pm 3.0$ & & \\
\hline 41.358 & 241.79 & $\mathrm{HCN}$ & $14-13$ & 41.356 & $876.0 \pm 11.1$ & 41.357 & $600.9 \pm 3.9$ & 41.358 & $16.5 \pm 3.6$ \\
\hline 41.561 & 240.61 & $\mathrm{HCN}$ & $v_{2}=1 ; 14-13 \mathrm{f}$ & 41.545 & $166.2 \pm 11.4$ & & & & \\
\hline 42.263 & 236.61 & ${ }^{12} \mathrm{CO}$ & $11-10$ & 42.263 & $3765.0 \pm 24.0$ & 42.265 & $2615.0 \pm 13.2$ & 42.253 & $2365.0 \pm 12.6$ \\
\hline 43.162 & 231.68 & $\mathrm{H}^{13} \mathrm{CN}$ & $15-14$ & 43.156 & $114.6 \pm 9.0$ & & & & \\
\hline 43.655 & 229.07 & $\mathrm{CCH}$ & $N=15-14$ & & & & & 43.649 & $29.7 \pm 3.0$ \\
\hline 44.073 & 226.90 & ${ }^{13} \mathrm{CO}$ & $12-11$ & 44.072 & $512.7 \pm 10.5$ & 44.078 & $262.8 \pm 3.3$ & 44.062 & $64.5 \pm 3.0$ \\
\hline 44.308 & 225.69 & $\mathrm{HCN}$ & $15-14$ & 44.303 & $852.3 \pm 10.2$ & 44.306 & $570.3 \pm 5.4$ & & \\
\hline 44.525 & 224.59 & $\mathrm{HCN}$ & $v_{2}=1 ; 15-14 \mathrm{f}$ & 44.523 & $133.5 \pm 22.8$ & & & & \\
\hline 44.919 & 222.62 & $\mathrm{CN}$ & $N=12-11 ; v=1$ & 44.903 & $20.1 \pm 9.3$ & & & & \\
\hline 45.318 & 220.66 & $\mathrm{HNC}$ & $15-14$ & 45.324 & $65.4 \pm 7.5$ & 45.323 & $160.5 \pm 3.9$ & & \\
\hline 46.098 & 216.93 & ${ }^{12} \mathrm{CO}$ & $12-11$ & 46.096 & $4356.0 \pm 15.3$ & 46.097 & $3009.0 \pm 100.5$ & 46.087 & $2370.0 \pm 9.3$ \\
\hline 46.428 & 215.39 & $\mathrm{HN}^{13} \mathrm{C}$ & $16-15$ & 46.443 & $32.7 \pm 16.2$ & 46.439 & $20.4 \pm 6.9$ & & \\
\hline 46.922 & 213.12 & $\mathrm{p}-\mathrm{H}_{2} \mathrm{O}$ & $v_{2}=1 ; 3_{21}-3_{12}$ & & & 46.918 & $7.5 \pm 4.8$ & & \\
\hline 47.053 & 212.53 & $\mathrm{O}-\mathrm{H}_{2} \mathrm{O}$ & $5_{23}-5_{14}$ & & & 47.049 & $43.8 \pm 6.6$ & & \\
\hline 47.256 & 211.61 & $\mathrm{HCN}$ & $16-15$ & 47.255 & $837.9 \pm 9.9$ & 47.255 & $570.0 \pm 7.5$ & & \\
\hline 47.487 & 210.58 & $\mathrm{HCN}$ & $v_{2}=1 ; 16-15 \mathrm{f}$ & & & 47.469 & $73.8 \pm 12.6$ & & \\
\hline 47.555 & 210.28 & $\mathrm{HCO}^{+}$ & $16-15$ & & & 47.555 & $73.2 \pm 11.7$ & 47.565 & $28.2 \pm 5.4$ \\
\hline 47.738 & 209.48 & ${ }^{13} \mathrm{CO}$ & $13-12$ & 47.737 & $488.1 \pm 16.2$ & 47.740 & $217.2 \pm 1.8$ & 47.726 & $80.4 \pm 6.3$ \\
\hline 48.333 & 206.90 & $\mathrm{HNC}$ & $16-15$ & & & 48.329 & $91.8 \pm 1.5$ & 48.337 & $20.7 \pm 6.3$ \\
\hline 48.655 & 205.53 & $\mathrm{CN}$ & $N=13-12 ; v=1$ & & & 48.658 & $118.5 \pm 3.6$ & & \\
\hline 48.738 & 205.18 & {$[\mathrm{~N} \mathrm{II}]$} & $2-1$ & & & & & 48.725 & $73.5 \pm 7.8$ \\
\hline 48.921 & 204.41 & $\mathrm{H} \mathrm{I}$ & $\mathrm{H} 16 \alpha$ & 48.924 & $212.4 \pm 22.5$ & 48.922 & $177.6 \pm 3.3$ & 48.918 & $102.3 \pm 7.2$ \\
\hline 49.117 & 203.60 & $\mathrm{CN}$ & $N=13-12 ; v=0$ & & & 49.121 & $219.9 \pm 4.5$ & & \\
\hline 49.932 & 200.27 & ${ }^{12} \mathrm{CO}$ & $13-12$ & 49.932 & $4329.0 \pm 27.3$ & 49.933 & $3387.0 \pm 15.3$ & 49.921 & $2252.0 \pm 9.6$ \\
\hline 50.203 & 199.19 & $\mathrm{HCN}$ & $17-16$ & 50.204 & $709.2 \pm 22.5$ & 50.205 & $552.3 \pm 6.6$ & & \\
\hline 50.521 & 197.94 & $\mathrm{HCO}^{+}$ & $17-16$ & & & 50.515 & $117.6 \pm 14.1$ & & \\
\hline 51.402 & 194.55 & ${ }^{13} \mathrm{CO}$ & $14-13$ & 51.393 & $444.9 \pm 22.5$ & 51.381 & $298.5 \pm 8.4$ & & \\
\hline
\end{tabular}


Table 2. Unidentified strong lines in the spectrum of AFGL 2688.

\begin{tabular}{|c|c|c|c|}
\hline $\bar{v}_{0}\left(\mathrm{~cm}^{-1}\right)$ & $\lambda_{0}(\mu \mathrm{m})$ & $F\left(\times 10^{-18} \mathrm{Wm}^{-2}\right)$ & Possible carriers \\
\hline 15.657 & 638.69 & $201.3 \pm 9.0$ & $\mathrm{NH}_{2}$ \\
\hline 15.762 & 634.44 & $139.5 \pm 8.7$ & $\mathrm{H}^{13} \mathrm{CN}$ v $2 ;{ }^{30} \mathrm{SiS}$ \\
\hline 15.931 & 627.71 & $116.1 \pm 8.4$ & $\mathrm{SiO} 11-10$ \\
\hline 16.547 & 604.34 & $193.8 \pm 6.3$ & $\mathrm{NH}_{2}$ \\
\hline 16.675 & 599.70 & $122.7 \pm 5.1$ & $\mathrm{H}^{13} \mathrm{CN} \mathrm{v1+v2}$ \\
\hline 16.856 & 593.26 & $304.8 \pm 12.0$ & $\mathrm{o}-\mathrm{SH}_{2}$ \\
\hline 16.998 & 588.30 & $193.8 \pm 21.9$ & $\mathrm{CH}_{2}$ \\
\hline 17.927 & 557.82 & $194.1 \pm 8.4$ & $\mathrm{CH}$ \\
\hline 18.766 & 532.88 & $122.1 \pm 3.9$ & $\mathrm{SiS} ; \mathrm{SiO} 13-12$ \\
\hline 18.945 & 527.84 & $195.0 \pm 3.9$ & $\mathrm{p}-\mathrm{SH}_{2}$ \\
\hline 19.440 & 514.40 & $159.9 \pm 3.6$ & \\
\hline 20.122 & 496.97 & $130.5 \pm 7.5$ & \\
\hline 21.208 & 471.52 & $201.6 \pm 12.0$ & \\
\hline 29.287 & 341.45 & $141.9 \pm 4.8$ & $\mathrm{HCN}$ v $2+2 \mathrm{v} 3 ; \mathrm{C}_{18} \mathrm{O}$ \\
\hline 31.479 & 317.67 & $134.7 \pm 20.4$ & \\
\hline 31.778 & 314.68 & $114.9 \pm 20.4$ & $\mathrm{NH}_{2}$ \\
\hline 32.665 & 306.14 & $150.3 \pm 7.8$ & $\mathrm{HCN} v_{2}=0$ \\
\hline 36.432 & 274.48 & $109.5 \pm 15.6$ & \\
\hline 38.842 & 257.45 & $105.9 \pm 4.8$ & \\
\hline 39.685 & 251.98 & $100.5 \pm 6.0$ & \\
\hline 47.456 & 210.72 & $101.7 \pm 72.3$ & $\mathrm{HCN} v_{2}=1$ \\
\hline 47.522 & 210.43 & $128.1 \pm 85.5$ & \\
\hline 49.589 & 201.66 & $146.1 \pm 27.0$ & \\
\hline 50.814 & 196.80 & $114.9 \pm 22.2$ & $\mathrm{HCN} 3 \mathrm{v} 2$ \\
\hline
\end{tabular}

Table 3. Unidentified lines in the spectrum of AFGL 618.

\begin{tabular}{llll}
\hline \hline $\bar{v}_{0}\left(\mathrm{~cm}^{-1}\right)$ & $\lambda_{0}(\mu \mathrm{m})$ & $F\left(\times 10^{-18} \mathrm{Wm}^{-2}\right)$ & Possible carriers \\
\hline 15.782 & 633.63 & $111.0 \pm 18.9$ & $\mathrm{H}^{13} \mathrm{CN} \mathrm{v} 2$ \\
16.472 & 607.09 & $129.3 \pm 4.8$ & \\
18.461 & 541.68 & $206.7 \pm 12.3$ & \\
19.444 & 514.30 & $116.1 \pm 5.4$ & $\mathrm{HCN}$ maser? \\
19.579 & 510.75 & $110.7 \pm 5.4$ & \\
27.396 & 365.02 & $150.3 \pm 2.7$ & \\
29.715 & 336.53 & $189.6 \pm 8.1$ & $\mathrm{HCN} \mathrm{2v2}$ \\
36.418 & 274.59 & $105.9 \pm 28.8$ & \\
\hline
\end{tabular}

Table 4. Unidentified lines in the spectrum of NGC 7027.

\begin{tabular}{llll}
\hline \hline $\bar{v}_{0}\left(\mathrm{~cm}^{-1}\right)$ & $\lambda_{0}(\mu \mathrm{m})$ & $F\left(\times 10^{-18} \mathrm{Wm}^{-2}\right)$ & Possible carriers \\
\hline 15.650 & 638.98 & $201.9 \pm 9.0$ & $\mathrm{NH}_{2}$ \\
16.983 & 588.82 & $128.1 \pm 20.1$ & $\mathrm{CH}_{2}$ \\
17.662 & 566.19 & $249.3 \pm 30.6$ & $\mathrm{NH}_{2}$ \\
17.880 & 559.28 & $101.1 \pm 10.8$ & $\mathrm{CH}$ \\
17.941 & 557.38 & $120.9 \pm 13.2$ & $\mathrm{H} 37 \epsilon$ \\
18.062 & 553.65 & $121.5 \pm 5.1$ & $\mathrm{H}^{+}, \mathrm{He}^{+}, \mathrm{C}^{+}, \mathrm{S}^{+} 28 \beta$ \\
18.939 & 528.01 & $181.8 \pm 11.1$ & $\mathrm{H}_{4} \delta ; \mathrm{p}^{+} \mathrm{SH}_{2}$ \\
20.101 & 497.49 & $109.8 \pm 16.2$ & $\mathrm{H}^{+}, \mathrm{He}^{+}, \mathrm{C}^{+}, \mathrm{S}^{+} 27 \beta$ \\
48.138 & 207.74 & $100.5 \pm 7.5$ & $\mathrm{NH}_{2}, \mathrm{H} 26 \epsilon$ \\
\hline
\end{tabular}

